\title{
WEAK SOLUTIONS TO THE NAVIER-STOKES EQUATIONS IN A Y-SHAPED DOMAIN
}

Abstract. We prove the existence of weak solutions to the Navier-Stokes equations describing the motion of a fluid in a Y-shaped domain.

1. Introduction. We consider the inflow-outflow problem in a reverse Y-shaped domain, with one inflow and two outflows. This can be treated as a simple model of the blood flow in veins or arteries. The motion of the fluid is described by the Navier-Stokes equations with boundary slip conditions. The domain $\Omega \subset \mathbb{R}^{3}$ is given by $\Omega=\Omega_{1} \cup \Omega_{2} \cup \Omega_{3}$ with the boundary $\partial \Omega=S=\sum_{i} S_{0}^{i} \cup S_{i}^{i}$ where $\Omega_{i}, i=1,2,3$, is a cylindrical type domain. To simplify the notation, we often omit the obvious index $i$ so that $S_{i}^{i} \equiv S_{i}$. We denote by $\bar{n}$ the unit outward vector normal to the boundary $S$ and by $\bar{\tau}_{j}, j=1,2$, vectors tangent to $S$. We introduce the velocity vector $v(x, t)=\left(v^{1}(x, t), v^{2}(x, t), v^{3}(x, t)\right)$ $\in \mathbb{R}^{3}$ with $v_{i}(x, t)=\left.v(x, t)\right|_{\Omega_{i}}$, the velocity defined on $\Omega_{i}$, and the pressure $p=p(x, t) \in \mathbb{R}^{1}$. The domain $\Omega$ and the velocity vectors are presented in Figure 1.

The problem reads

$$
\begin{array}{ll}
v_{t}+v \cdot \nabla v-\operatorname{div} \mathbb{T}(v, p)=f & \text { in } \Omega^{T}=\Omega \times(0, T), \\
\operatorname{div} v=0 & \text { in } \Omega^{T}, \\
\left.v\right|_{t=0}=v(0), & \\
\left.v \cdot \bar{n}\right|_{S_{0}^{i}}=0, & \\
\left.v \cdot \bar{n}\right|_{S_{1}}=-a_{1}, &
\end{array}
$$

2000 Mathematics Subject Classification: 35Q35, 76D03, 76D05.

Key words and phrases: Navier-Stokes equations, Y-shaped domain, inflow-outflow problem, slip boundary conditions, weak solutions.

Research supported by KBN grant no. 1 P03A 02130. 


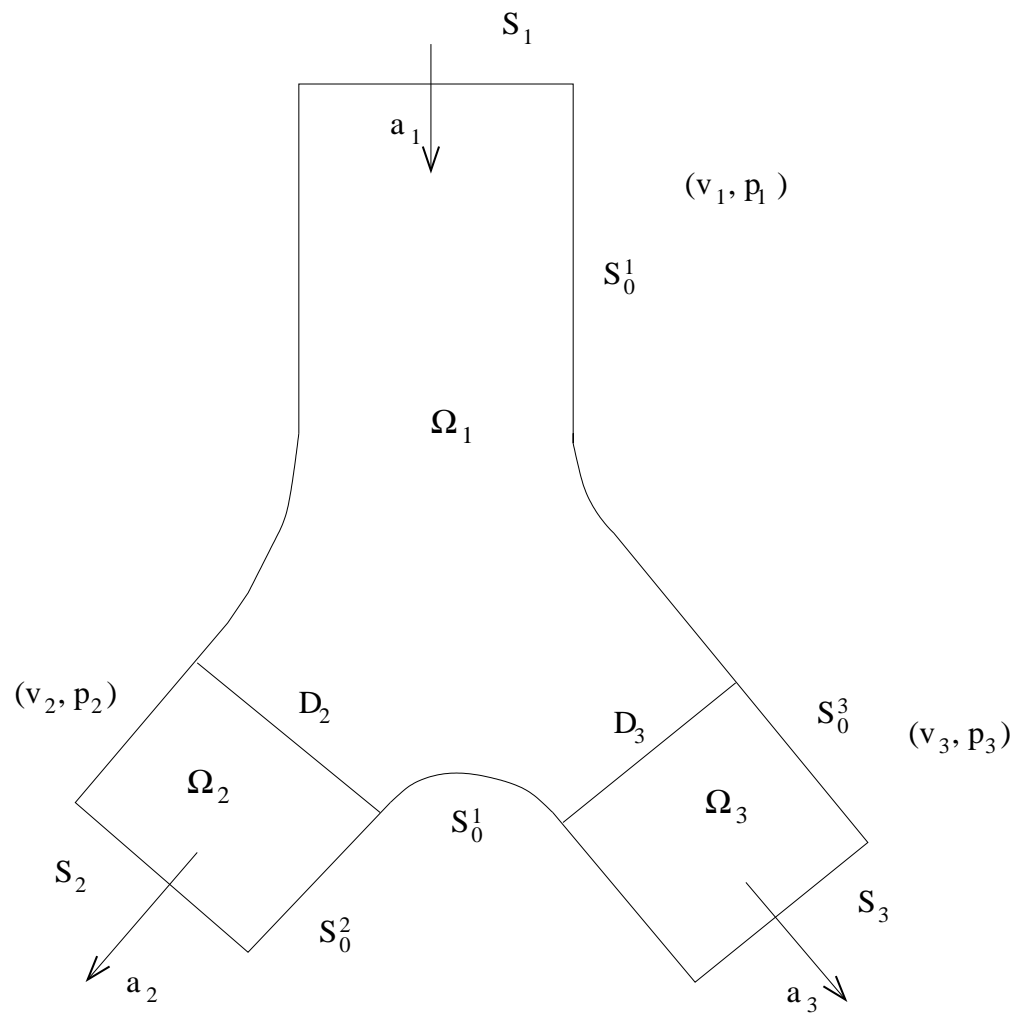

Fig. 1. Y-shaped domain

[cont.]

$$
\left.v \cdot \bar{n}\right|_{S_{i}}=a_{i}, \quad i=2,3,
$$

$$
\nu \bar{n} \cdot \mathbb{D}(v) \cdot \bar{\tau}_{j}+\gamma v \cdot \bar{\tau}_{j}=0, \quad j=1,2, \quad \text { on } S_{0}^{i},
$$

$$
\bar{n} \cdot \mathbb{D}(v) \cdot \bar{\tau}_{j}=0, \quad j=1,2, \quad \text { on } S_{i}, i=1,2,3,
$$

where $f=f(x, t)=\left(f^{1}(x, t), f^{2}(x, t), f^{3}(x, t)\right) \in \mathbb{R}^{3}$ is the external force, $\nu$ is the constant viscosity coefficient, $\gamma>0$ is the slip coefficient, and the stress tensor $\mathbb{T}$ and the dilatation tensor $\mathbb{D}$ are given as

$$
\mathbb{D}(v)=\left\{v_{, x_{j}}^{i}+v_{, x_{i}}^{j}\right\}_{i, j=1,2,3}, \quad \mathbb{T}(v, p)=\nu \mathbb{D}(v)-p I .
$$

The inflow $a_{1}$ and outflows $a_{2}, a_{3}$ satisfy the compatibility condition

$$
\int_{S_{1}} a_{1}=\int_{S_{2}} a_{2}+\int_{S_{3}} a_{3} .
$$

We set $\bar{n}_{i}=\left.\bar{n}\right|_{\Omega_{i}}$. We define the artificial boundaries $D_{i}=\Omega_{1} \cap \Omega_{i}, i=2,3$.

Then

$$
\begin{aligned}
& v_{1}=v_{i}, \\
& \bar{n}_{1} \cdot \mathbb{T}\left(v_{1}, p_{1}\right)=\bar{n}_{1} \cdot \mathbb{T}\left(v_{i}, p_{i}\right) \quad \text { on } D_{i}, i=2,3, j=1,2 .
\end{aligned}
$$


In Section 2, we prove some a priori energy type estimates. This is motivated by considerations from [Z1]. Section 3 is devoted to the proof of existence of weak solutions to the problem (1.1) by the Galerkin method (see [L, Chapter 6, Section 7]). The last part is the Appendix where the properties of solutions in the neighborhood of the transmission sections $D_{2}$ and $D_{3}$ are examined.

2. Problem reformulation and a priori estimates. To obtain energy type estimates we need to work with a function $v$ which satisfies the homogeneous Dirichlet boundary condition. To reformulate the problem (1.1) we introduce a new function $\alpha$ satisfying

$$
\left.\alpha_{1} \cdot \bar{n}_{1}\right|_{S_{1}}=-a_{1},\left.\quad \alpha_{i} \cdot \bar{n}_{1}\right|_{S_{i}}=-a_{i}, \quad i=2,3,
$$

and next, we define functions $u_{i}$ on $\Omega_{i}$ by

$$
u_{i}=v_{i}-\alpha_{i}, \quad i=1,2,3 .
$$

Thus we have

$$
\operatorname{div} u_{i}=-\operatorname{div} \alpha_{i},\left.\quad u_{i} \cdot \bar{n}_{i}\right|_{S_{i}}=0 .
$$

Let $\varphi=\left(\varphi_{1}, \varphi_{2}, \varphi_{3}\right)$ be a solution to the problem

$$
\begin{array}{ll}
\Delta \varphi_{i}=-\operatorname{div} \alpha_{i} & \text { in } \Omega_{i}, \\
\bar{n}_{i} \cdot \nabla \varphi_{i}=0 & \text { on } S_{i} \text { and } S_{0}^{i}, \\
\int_{\Omega_{i}} \varphi_{i} d x=0, & \\
\varphi_{1}=\varphi_{i} & \text { on } D_{i}, i=2,3, \\
\frac{\partial}{\partial n_{1}} \varphi_{1}=\frac{\partial}{\partial n_{1}} \varphi_{i} & \text { on } D_{i}, i=2,3,
\end{array}
$$

where $n_{i}$ is the curvilinear coordinate along the curve tangent to $\bar{n}_{i}$, $i=1,2,3$. We claim

Lemma 2.1. For every extension function $\alpha$ such that $\alpha_{i} \in H^{1}\left(\Omega_{i}\right)$, $i=1,2,3$, there exists a solution $\varphi=\left(\varphi_{1}, \varphi_{2}, \varphi_{3}\right)$ to the problem (2.1) and the following bound holds:

$$
\sum_{i=1}^{3}\left\|\nabla \varphi_{i}\right\|_{H^{2}\left(\Omega_{i}\right)} \leq c \sum_{i=1}^{3} \int\left\|\alpha_{i}\right\|_{H^{1}\left(\Omega_{i}\right)} .
$$

For convenience of the reader, we sketch the proof of this technical result in the Appendix.

Therefore, we can define new functions

$$
w_{i}=v_{i}-\alpha_{i}-\nabla \varphi_{i} \equiv v_{i}-\delta_{i}
$$


satisfying the following system:

$$
\begin{aligned}
& w_{i, t}+w_{i} \cdot \nabla w_{i}+w_{i} \cdot \nabla \delta_{i}+\delta_{i} \cdot \nabla w_{i}-\operatorname{div} \mathbb{T}\left(w_{i}, p_{i}\right) \\
& \quad=f_{i}-\delta_{i, t}-\delta_{i} \cdot \nabla \delta_{i}+\nu \operatorname{div} \mathbb{D}\left(\delta_{i}\right) \equiv F_{i} \quad \operatorname{in} \Omega_{i}, \\
& \operatorname{div} w_{i}=0
\end{aligned}
$$

and the transmission conditions

$$
w_{1}=w_{i} \quad \text { and } \quad \frac{\partial}{\partial n_{1}} w_{1}=\frac{\partial}{\partial n_{1}} w_{i} \quad \text { on } D_{i}, i=2,3 .
$$

Now, we introduce weak solutions to $(2.3)-(2.4)$.

Definition 2.1. A weak solution to $(2.3)-(2.4)$ is a triple $\left(w_{1}, w_{2}, w_{3}\right)$ satisfying the identities

$$
\sum_{i=1}^{3}\left(\int_{\Omega_{i}^{T}} w_{i, t} \varphi d x d t+\int_{\Omega_{i}^{T}} H\left(w_{i}\right) \varphi d x d t+\nu \int_{\Omega_{i}^{T}} \mathbb{D}\left(w_{i}\right) \mathbb{D}(\varphi) d x d t\right.
$$

$\left.+\gamma \sum_{j=1}^{2} \int_{S_{0}^{T}} w_{i} \cdot \bar{\tau}_{j} \varphi \cdot \bar{\tau}_{j} d S_{0}^{i} d t-\sum_{j=1}^{2} \sum_{\sigma=0, i} \int_{S_{\sigma}^{i T}} B_{\sigma j}^{i} \varphi \cdot \bar{\tau}_{j} d S_{\sigma}^{i}\right)=\sum_{i=1}^{3} \int_{\Omega_{i}^{T}} F_{i} \cdot \varphi d x d t$

where $H(w)=w \cdot \nabla w+w \cdot \nabla \delta+\delta \cdot \nabla w$, for any sufficiently smooth function $\varphi$ with $\operatorname{div} \varphi=0,\left.\varphi \cdot \bar{n}\right|_{S}=0$.

We introduce some useful notation:

$$
\begin{array}{ll}
|u|_{p, Q}=\sum_{i=1}^{3}\|u\|_{L_{p}\left(Q_{i}\right)}, & Q \in\left\{\Omega^{T}, S^{T}, \Omega, S\right\}, p \in[1, \infty], \\
\|u\|_{s, Q}=\sum_{i=1}^{3}\|u\|_{H^{s}\left(Q_{i}\right)}, & Q \in\{\Omega, S\}, s \in \mathbb{R}_{+} \cup\{0\}, \\
|u|_{p, q, Q^{T}}=\sum_{i=1}^{3}\|u\|_{L_{q}\left(0, T ; L_{p}\left(Q_{i}\right)\right)}, & Q \in\{\Omega, S\}, p, q \in[1, \infty],
\end{array}
$$


and a space natural for the study of the Navier-Stokes equations:

$V_{2}^{0}\left(\Omega^{T}\right)=\left\{u:\|u\|_{V_{2}^{0}\left(\Omega^{T}\right)}=\operatorname{essip}_{t \in(0, T)}\|u\|_{L_{2}(\Omega)}+\left(\int_{0}^{T}\|\nabla u\|_{L_{2}(\Omega)}^{2} d t\right)^{1 / 2}<\infty\right\}$.

We will need the following result:

Lemma 2.2 (Korn inequality). Assume that

$$
E_{\Omega}(w)=\sum_{i, j=1}^{3} \int_{\Omega}\left(w_{x_{j}}^{i}+w_{x_{i}}^{j}\right)^{2} d x<\infty
$$

and

$$
\sum_{j=1}^{2}\left|w \cdot \bar{\tau}_{j}\right|_{2, S_{0}}^{2}<\infty,\left.\quad w \cdot \bar{n}\right|_{S}=0,\left.\quad \operatorname{div} w\right|_{\Omega}=0 .
$$

Then there exists a constant $c$ independent of $w$ such that

$$
\|w\|_{H^{1}(\Omega)}^{2} \leq c\left(E_{\Omega}(w)+\sum_{j=1}^{2}\left|w \cdot \bar{\tau}_{j}\right|_{L_{2}\left(S_{0}\right)}^{2}\right) \equiv c E .
$$

Proof. We have

$$
\begin{aligned}
E_{\Omega}(w) & =2 \sum_{i, j=1}^{3}\left(\int_{\Omega}\left(w_{x_{j}}^{i}\right)^{2} d x+\int_{\Omega} w_{x_{j}}^{i} \cdot w_{x_{i}}^{j} d x\right) \\
& =2 \sum_{i, j=1}^{3}\left(\int_{\Omega}\left(w_{x_{j}}^{i}\right)^{2} d x+\int_{\Omega}\left(w_{x_{j}}^{i} \cdot w^{j}\right)_{x_{i}} d x\right) \\
& =2 \sum_{i, j=1}^{3}\left(\int_{\Omega}\left(w_{x_{j}}^{i}\right)^{2} d x+\int_{S} w_{x_{j}}^{i} \cdot w^{j} \cdot n_{i} d S\right) \\
& =2 \sum_{i, j=1}^{3}\left(\int_{\Omega}\left(w_{x_{j}}^{i}\right)^{2} d x-\int_{S} w^{i} \cdot w^{j} \cdot n_{i, x_{j}} d S\right),
\end{aligned}
$$

where $\int_{\Omega} f$ denotes $\sum_{i=1}^{3} \int_{\Omega_{i}} f_{i}$. This implies

$$
|\nabla w|_{2, \Omega}^{2} \leq c E
$$

For a non-axially symmetric function $w$ we can use the results in [Z2] to find that

$$
|w|_{2, \Omega}^{2} \leq \delta|\nabla w|_{2, \Omega}^{2}+M(\delta) E_{\Omega}(w)
$$

so that (2.9) and (2.10) yield (2.8).

We will show the following a priori estimate for $w$. 
Lemma 2.3. Assume that $a_{1} \in L_{6}\left(0, T ; L_{3}\left(S_{1}\right)\right), \nabla \alpha \in L_{2}\left(0, T ; L_{3}(\Omega)\right)$ and $w_{i}(0) \in L_{2}\left(\Omega_{i}\right), i=1,2,3$. Let

$$
\Gamma^{2}(t)=|f|_{6 / 5, \Omega}^{2}+\left|\alpha_{t}\right|_{6 / 5, \Omega}^{2}+\left|\nabla \alpha_{t}\right|_{6 / 5, \Omega}^{2}+|\alpha|_{2, S_{0}}^{2}+|\nabla \alpha|_{2, \Omega}^{2}\left(1+|\alpha|_{W_{3}^{1}(\Omega)}^{2}\right)
$$

with

$$
\int_{0}^{T} \Gamma^{2}(t) d t<\infty
$$

Then

$$
|w|_{V_{2}^{0}\left(\Omega^{t}\right)}^{2} \leq c e^{c\left(\left|a_{1}\right|_{3,6, S_{1}^{t}}^{6}+|\nabla \alpha|_{3,2, \Omega^{t}}^{2}\right)}\left(\int_{0}^{t} \Gamma^{2}\left(t^{\prime}\right) d t^{\prime}+|w(0)|_{2, \Omega}^{2}\right) .
$$

Proof. With $\varphi=w$ and $\left.w \cdot \bar{n}\right|_{S}=0$ we get by definition of weak solutions

$$
\begin{aligned}
& \sum_{i=1}^{3}\left(\frac{1}{2} \frac{d}{d t}\left|w_{i}\right|_{2, \Omega_{i}}^{2}+\int_{\Omega_{i}}\left|\delta_{i} \cdot \nabla w_{i} \cdot w_{i}+w_{i} \cdot \nabla \delta_{i} \cdot w_{i}\right| d x+\nu\left|\mathbb{D}\left(w_{i}\right)\right|_{2, \Omega_{i}}^{2}\right. \\
& \left.+\gamma\left|w_{i} \cdot \bar{\tau}_{j}\right|_{2, S_{0}^{i}}^{2}\right)=\sum_{i=1}^{3}\left(\sum_{j=1}^{2} \sum_{\sigma=0, i} \int_{S_{\sigma}^{i}} B_{\sigma j}^{i} w_{i} \cdot \bar{\tau}_{j} d S_{\sigma}^{i}+\int_{\Omega_{i}} F_{i} \cdot w_{i} d x\right) .
\end{aligned}
$$

Now, we analyze the second term on the l.h.s. We have

$$
\begin{aligned}
\int_{\Omega_{i}} \delta_{i} \cdot \nabla w_{i} \cdot w_{i} d x & =\int_{\Omega_{i}}\left(\alpha_{i}+\nabla \varphi_{i}\right) \cdot \nabla w_{i} \cdot w_{i} d x \\
& =\int_{\Omega_{i}} \alpha_{i} \cdot \nabla w_{i} \cdot w_{i} d x+\int_{\Omega_{i}} \nabla \varphi_{i} \cdot \nabla w_{i} \cdot w_{i} d x \equiv I_{1}+I_{2}
\end{aligned}
$$

so that

$$
\begin{aligned}
I_{1}\left(w_{i}\right) & =\frac{1}{2} \int_{\Omega_{i}} \alpha_{i} \cdot \nabla\left(w_{i}^{2}\right) d x \\
& =\frac{1}{2} \int_{\Omega_{i}} \operatorname{div}\left(\alpha_{i} w_{i}^{2}\right) d x-\frac{1}{2} \int_{\Omega_{i}} \operatorname{div} \alpha_{i} \cdot w_{i}^{2} d x \equiv I_{1}^{a}+I_{1}^{b} .
\end{aligned}
$$

Next, we calculate

$$
\begin{aligned}
I_{1}^{a}\left(w_{1}\right) & =-\frac{1}{2} \int_{S_{1}} a_{1} w_{i}^{2} d S_{1}+\frac{1}{2} \int_{D_{2}} \alpha \cdot \bar{n}_{1} w_{2}^{2} d D_{2}+\frac{1}{2} \int_{D_{3}} \alpha \cdot \bar{n}_{1} w_{3}^{2} d D_{3}, \\
I_{1}^{a}\left(w_{i}\right) & =\frac{1}{2} \int_{S_{i}} a_{i} w_{i}^{2} d S_{i}-\frac{1}{2} \int_{D_{i}} \alpha \cdot \bar{n}_{1} w_{i}^{2} d D_{i}, \quad i=2,3 .
\end{aligned}
$$

Thus,

$$
\sum_{i=1}^{3} I_{1}^{a}\left(w_{i}\right)=\frac{1}{2}\left(-\int_{S_{1}} a_{1} w_{1}^{2} d S_{1}+\int_{S_{2}} a_{2} w_{2}^{2} d S_{2}+\int_{S_{3}} a_{3} w_{3}^{2} d S_{3}\right),
$$


and we can estimate

$$
-\sum_{i=1}^{3} I_{1}^{a}\left(w_{i}\right) \leq \varepsilon_{1}^{a}\left\|w_{1}\right\|_{1, \Omega_{1}}^{2}+c\left(1 / \varepsilon_{1}^{a}\right)\left|a_{1}\right|_{3, S_{1}}^{6}\left|w_{1}\right|_{2, \Omega_{1}}^{2}
$$

For $I_{1}^{b}$ we have

$$
\left|\sum_{i=1}^{3} I_{1}^{b}\left(w_{i}\right)\right| \leq \varepsilon_{1}^{b}|w|_{6, \Omega}^{2}+c\left(1 / \varepsilon_{1}^{b}\right)|\nabla \alpha|_{3, \Omega}^{2}|w|_{2, \Omega}^{2}
$$

So

$$
\left|\sum_{i=1}^{3} I_{1}\left(w_{i}\right)\right| \leq \varepsilon_{1}\left(|w|_{6, \Omega}^{2}+\|w\|_{1, \Omega}^{2}\right)+c\left(1 / \varepsilon_{1}\right)\left(\left|a_{1}\right|_{3, S_{1}}^{6}+|\nabla \alpha|_{3, \Omega}^{2}\right)|w|_{2, \Omega}^{2} .
$$

Also we obtain

$$
\sum_{i=1}^{3} I_{2}\left(w_{i}\right)=\frac{1}{2} \sum_{i=1}^{3} \int_{\Omega_{i}} \nabla \varphi_{i} \cdot \nabla\left(w_{i}^{2}\right)=-\frac{1}{2} \sum_{i=1}^{3} \int_{\Omega_{i}} \Delta \varphi_{i} w_{i}^{2}=\frac{1}{2} \sum_{i=1}^{3} \int_{\Omega_{i}} \operatorname{div} \alpha_{i} w_{i}^{2} .
$$

Consequently,

$$
\left|\sum_{i=1}^{3} I_{2}\left(w_{i}\right)\right| \leq \varepsilon_{2}|w|_{6, \Omega}^{2}+c\left(1 / \varepsilon_{2}\right)|\nabla \alpha|_{3, \Omega}^{2}|w|_{2, \Omega}^{2}
$$

Next, we consider the expression

$$
\int_{\Omega_{i}} w_{i} \cdot \nabla \delta_{i} \cdot w_{i}=\int_{\Omega_{i}} w_{i} \cdot \nabla \alpha \cdot w_{i}+\int_{\Omega_{i}} w_{i} \cdot \nabla\left(\nabla \varphi_{i}\right) \cdot w_{i} \equiv I_{3}+I_{4}
$$

with

$$
\begin{aligned}
& \left|\sum_{i=1}^{3} I_{3}\left(w_{i}\right)\right| \leq \varepsilon_{3}|w|_{6, \Omega}^{2}+c\left(1 / \varepsilon_{3}\right)|\nabla \alpha|_{3, \Omega}^{2}|w|_{2, \Omega}^{2}, \\
& \left|\sum_{i=1}^{3} I_{4}\left(w_{i}\right)\right| \leq \varepsilon_{4}|w|_{6, \Omega}^{2}+c\left(1 / \varepsilon_{4}\right)|\nabla \alpha|_{3, \Omega}^{2}|w|_{2, \Omega}^{2} .
\end{aligned}
$$

We sum (2.12) over $i=1,2,3$, and use the above estimates. Then, we use the imbedding inequality

$$
|u|_{6, \Omega} \leq c\|u\|_{W_{2}^{1}(\Omega)},
$$

and the Korn inequality (2.8):

$$
\|w\|_{H^{1}(\Omega)} \leq c\left(\int_{\Omega_{i}} \mathbb{D}(w)^{2}+\sum_{j=1}^{2}\left|w \cdot \bar{\tau}_{j}\right|_{2, S_{0}}^{2}\right),
$$

to obtain 


$$
\begin{aligned}
\frac{1}{2} \frac{d}{d t}|w|_{2, \Omega}^{2} & +|w|_{H^{1}(\Omega)}^{2} \\
& \leq c A|w|_{2, \Omega}^{2}+\int_{\Omega} F \cdot w+\sum_{i=1}^{3} \sum_{j=1}^{2} \sum_{\sigma=0, i} \int_{S_{\sigma}^{i}} B_{\sigma, j}^{i} w_{i} \cdot \bar{\tau}_{j} d S_{\sigma}^{i} \\
& \equiv c A|w|_{2, \Omega}^{2}+J
\end{aligned}
$$

where $A=\left|a_{1}\right|_{3, S_{1}}^{6}+|\nabla \alpha|_{3, \Omega}^{2}$. We now deal with the r.h.s. of the above inequality. The last two terms have the form

$$
\begin{aligned}
J= & \sum_{i=1}^{3} \sum_{j=1}^{2} \int_{\Omega_{i}}\left(f_{i}-\delta_{i, t}-\delta_{i} \cdot \nabla \delta_{i}\right) w_{i} d x+\nu \int_{\Omega_{i}} \operatorname{div} \mathbb{D}\left(\delta_{i}\right) \cdot w_{i} d x \\
& -\int_{S_{0}^{i}}\left(\nu \bar{n}_{i} \mathbb{D}\left(\delta_{i}\right) \bar{\tau}_{j} w_{i} \cdot \bar{\tau}_{j}+\gamma \delta_{i} \cdot \bar{\tau}_{j} \cdot w_{i} \cdot \bar{\tau}_{j}\right) d S_{0}^{i}-\int_{S_{i}} \nu \bar{n}_{i} \mathbb{D}\left(\delta_{i}\right) \bar{\tau}_{j} w_{i} \cdot \bar{\tau}_{j} d S_{i} .
\end{aligned}
$$

To simplify we only study the second term of $J$ :

$$
\begin{aligned}
\int_{\Omega_{1}} \operatorname{div} \mathbb{D}\left(\delta_{1}\right) \cdot w_{1}= & \sum_{\sigma=0,1} \sum_{j=1}^{2} \int_{S_{\sigma}^{1}} \bar{n}_{1} \mathbb{D}\left(\delta_{1}\right) \bar{\tau}_{j} w_{1} \cdot \bar{\tau}_{j}-\int_{\Omega_{1}} \mathbb{D}\left(\delta_{1}\right) \cdot \mathbb{D}\left(w_{1}\right) \\
& +\sum_{k=2,3} \sum_{j=1}^{2} \int_{D_{k}} \bar{n}_{1} \mathbb{D}\left(\delta_{k}\right) \bar{\tau}_{j} w_{k} \cdot \bar{\tau}_{j}, \\
\int_{\Omega_{i}} \operatorname{div} \mathbb{D}\left(\delta_{i}\right) \cdot w_{i}= & \sum_{\sigma=0, i} \sum_{j=1}^{2} \int_{S_{\sigma}^{i}} \bar{n}_{i} \mathbb{D}\left(\delta_{i}\right) \bar{\tau}_{j} w_{i} \cdot \bar{\tau}_{j}-\int_{\Omega_{i}} \mathbb{D}\left(\delta_{i}\right) \cdot \mathbb{D}\left(w_{i}\right) \\
& +\sum_{j=1}^{2} \int_{D_{i}} \bar{n}_{i} \mathbb{D}\left(\delta_{i}\right) \bar{\tau}_{j} w_{i} \cdot \bar{\tau}_{j} \quad \\
= & \sum_{\sigma=0, i} \sum_{j=1}^{2} \int_{S_{\sigma}^{i}} \bar{n}_{i} \mathbb{D}\left(\delta_{i}\right) \bar{\tau}_{j} w_{i} \cdot \bar{\tau}_{j}-\int_{\Omega_{i}} \mathbb{D}\left(\delta_{i}\right) \cdot \mathbb{D}\left(w_{i}\right) \\
& -\sum_{j=1}^{2} \int_{D_{i}} \bar{n}_{1} \mathbb{D}\left(\delta_{i}\right) \bar{\tau}_{j} w_{i} \cdot \bar{\tau}_{j}, \quad i=2,3,
\end{aligned}
$$

to obtain

$$
\begin{aligned}
J & =\sum_{i=1}^{3}\left(\int_{\Omega}\left(f_{i}-\delta_{i, t}-\delta_{i} \cdot \nabla \delta_{i}\right) \cdot w_{i}-\gamma \int_{S_{0}^{i}} \delta_{i} \cdot \bar{\tau}_{j} \cdot w_{i} \cdot \bar{\tau}_{j} d S_{0}^{i}-\nu \int_{\Omega} \mathbb{D}\left(\delta_{i}\right) \cdot \mathbb{D}\left(w_{i}\right)\right) \\
& \equiv \sum_{i=1}^{3}\left(J_{1}^{i}+J_{2}^{i}+J_{3}^{i}\right) \equiv J_{1}+J_{2}+J_{3} .
\end{aligned}
$$


Then

$$
\left|J_{1}\right| \leq \varepsilon_{5}|w|_{6, \Omega}^{2}+c\left(1 / \varepsilon_{5}\right)\left(|f|_{6 / 5, \Omega}^{2}+\left|\delta_{t}\right|_{6 / 5, \Omega}^{2}+|\delta \cdot \nabla \delta|_{6 / 5, \Omega}^{2}\right),
$$

where

$$
\begin{aligned}
\left|\delta_{t}\right|_{6 / 5, \Omega} & \leq\left|\alpha_{t}\right|_{6 / 5, \Omega}+\left|\nabla \varphi_{t}\right|_{6 / 5, \Omega} \leq\left|\alpha_{t}\right|_{6 / 5, \Omega}+\left|\int_{\Omega} \nabla G \nabla \alpha_{t}\right|_{6 / 5, \Omega} \\
& \leq c\left(\left|\alpha_{t}\right|_{6 / 5, \Omega}+\left|\nabla \alpha_{t}\right|_{6 / 5, \Omega}\right),
\end{aligned}
$$

and $G$ is the Green function for the problem for $\varphi$. Similarly,

$$
|\delta \cdot \nabla \delta|_{6 / 5, \Omega} \leq|\delta|_{3, \Omega}|\nabla \delta|_{2, \Omega} \leq c|\alpha|_{W_{3}^{1}(\Omega)}|\nabla \alpha|_{2, \Omega} .
$$

We examine $J_{2}$ and $J_{3}$ to get

$$
\begin{aligned}
\left|J_{2}\right| & \leq \varepsilon_{6}|w|_{H^{1}(\Omega)}^{2}+c\left(1 / \varepsilon_{6}\right)\left(|\alpha|_{2, S_{0}}^{2}+\sum_{j=1}^{2}\left|\bar{\tau}_{j} \cdot \nabla \varphi\right|_{2, S_{0}}^{2}\right) \\
& \leq \varepsilon_{6}|w|_{H^{1}}^{2}+c\left(1 / \varepsilon_{6}\right)\left(|\alpha|_{2, S_{0}}^{2}+|\nabla \alpha|_{2, \Omega}^{2}\right), \\
\left|J_{3}\right| & \leq \varepsilon_{7}|w|_{H^{1}(\Omega)}^{2}+c\left(1 / \varepsilon_{7}\right)|\mathbb{D}(\delta)|_{2, \Omega}^{2} \\
& \leq \varepsilon_{7}|w|_{H^{1}(\Omega)}^{2}+c\left(1 / \varepsilon_{7}\right)\left(|\nabla \alpha|_{2, \Omega}+|\nabla \nabla \varphi|_{2, \Omega}\right)^{2} \\
& \leq \varepsilon_{7}|w|_{H^{1}(\Omega)}^{2}+c\left(1 / \varepsilon_{7}\right)|\nabla \alpha|_{2, \Omega}^{2} .
\end{aligned}
$$

The above estimates yield

$$
\begin{aligned}
|J| \leq & \varepsilon|w|_{H^{1}(\Omega)}+c(1 / \varepsilon)\left[|f|_{6 / 5, \Omega}^{2}+\left|\alpha_{t}\right|_{6 / 5, \Omega}^{2}+\left|\nabla \alpha_{t}\right|_{6 / 5, \Omega}^{2}+|\alpha|_{2, S_{0}}^{2}\right. \\
& \left.+|\nabla \alpha|_{2, \Omega}^{2}\left(1+|\alpha|_{W_{3}^{1}(\Omega)}^{2}\right)\right] \\
= & \varepsilon|w|_{H^{1}(\Omega)}+c(1 / \varepsilon) \Gamma^{2}(t) .
\end{aligned}
$$

Then from (2.13) we obtain

$$
\frac{1}{2} \frac{d}{d t}|w|_{2, \Omega}^{2}+|w|_{H^{1}(\Omega)}^{2} \leq c\left(A|w|_{2, \Omega}^{2}+\Gamma^{2}(t)\right) .
$$

If we set $A(t)=\left|a_{1}\right|_{3,6, S_{1}^{t}}^{6}+|\nabla \alpha|_{3,2, \Omega^{t}}^{2}$, this can be rewritten as

$$
\frac{d}{d t}\left(|w|_{2, \Omega}^{2} e^{-c A(t)}\right)+|w|_{H^{1}(\Omega)}^{2} e^{-c A(t)} \leq c \Gamma^{2}(t) e^{-c A(t)}
$$

and integrated in time:

$$
\begin{aligned}
|w(t)|_{2, \Omega}^{2}+e^{c A(t)} \int_{0}^{t}\left|w\left(t^{\prime}\right)\right|_{H^{1}(\Omega)}^{2} e^{-c A\left(t^{\prime}\right)} d t^{\prime} & \\
& \leq c e^{c A(t)}\left(\int_{0}^{t} \Gamma^{2}\left(t^{\prime}\right) e^{-c A\left(t^{\prime}\right)} d t^{\prime}+|w(0)|_{2, \Omega}^{2}\right) .
\end{aligned}
$$


We can estimate the r.h.s. and simplify as follows:

$$
|w(t)|_{2, \Omega}^{2}+\int_{0}^{t}\left|w\left(t^{\prime}\right)\right|_{H^{1}(\Omega)}^{2} d t^{\prime} \leq c e^{c A(t)}\left(\int_{0}^{t} \Gamma^{2}\left(t^{\prime}\right) d t^{\prime}+|w(0)|_{2, \Omega}^{2}\right) .
$$

Omitting the first term on the l.h.s. we get

$$
\int_{0}^{t}\left|w\left(t^{\prime}\right)\right|_{H^{1}(\Omega)}^{2} d t^{\prime} \leq c e^{c A(t)}\left(\int_{0}^{t} \Gamma^{2}\left(t^{\prime}\right) d t^{\prime}+|w(0)|_{2, \Omega}^{2}\right) .
$$

On the other hand, we can omit the second term in (2.15) to obtain

$$
\frac{d}{d t}\left(|w|_{2, \Omega}^{2} e^{-c A(t)}\right) \leq c \Gamma^{2}(t),
$$

and integrate in time to get the estimate for $|w|_{2, \Omega}$. Together with (2.16) this gives the result.

We have $v=w+\delta=w+\alpha+\nabla \varphi$ where

$$
\begin{aligned}
|\delta|_{V_{2}^{0}\left(\Omega^{T}\right)}^{2} & \leq|\delta|_{2, \infty, \Omega^{T}}^{2}+\int_{0}^{T}\left\|\delta\left(t^{\prime}\right)\right\|_{1, \Omega}^{2} d t^{\prime} \\
& \leq|\alpha|_{2, \infty, \Omega^{T}}^{2}+|\nabla \alpha|_{2, \infty, \Omega^{T}}^{2}+\int_{0}^{T}\left\|\alpha\left(t^{\prime}\right)\right\|_{1, \Omega}^{2} d t^{\prime} .
\end{aligned}
$$

Thus, we have the following corollary:

LEMMA 2.4. Let the assumptions of Lemma 2.3 be satisfied and

$$
\Lambda(T)=c\left(|\alpha|_{2, \infty, \Omega^{T}}^{2}+|\nabla \alpha|_{2, \infty, \Omega^{T}}^{2}\right)+\int_{0}^{T}\left\|\alpha\left(t^{\prime}\right)\right\|_{1, \Omega}^{2} d t^{\prime}<\infty .
$$

Then

$$
|v|_{V_{2}^{0}\left(\Omega^{T}\right)}^{2} \leq c e^{c\left(\left|a_{1}\right|_{3,6, S_{1}^{T}}^{6}+|\nabla \alpha|_{3,2, \Omega^{T}}^{2}\right)}\left(\int_{0}^{T} \Gamma^{2}\left(t^{\prime}\right) d t^{\prime}+|v(0)|_{2, \Omega}^{2}\right)+\Lambda(T) .
$$

3. Weak solutions to (2.3). In this section, we follow the ideas from [L, Chapter 6, Section 7]. We will use the Galerkin method to prove the existence of weak solutions to the problem (2.3). Namely, we introduce the sequence of approximating functions $w_{N}$ given as

$$
w^{N}(x, t)=\sum_{k=1}^{N} C_{k N}(t) a^{k}(x),
$$

where $\left\{a^{k}\right\}_{k=1}^{\infty}$ is a system of orthogonal functions in $L_{2}(\Omega) \cap J_{2}^{0}(\Omega)$. Here, $J_{2}^{0}(\Omega)=\left\{f \in H^{1}(\Omega): \operatorname{div} f=0\right\}$ and $\left\{a^{k}\right\}_{k=1}^{\infty}$ is a fundamental system in $H^{1}(\Omega)$ with $\sup _{x \in \Omega}\left|a^{k}(x)\right|<\infty, \sup _{x \in \partial \Omega}\left|a^{k}(x)\right|<\infty$. The coefficients 
$C_{k N}(0)$ are defined by

$$
\left.C_{k N}\right|_{t=0}=\left(w_{0}, a_{k}\right), \quad k=1, \ldots, N,
$$

and the functions $w^{N}$ satisfy the following system with test functions $a^{k}$ :

$$
\begin{aligned}
& \sum_{i=1}^{3}\left\{\int _ { \Omega _ { i } } \left(\frac{1}{2} \frac{d}{d t} w_{i}^{N} a^{k}+w_{i}^{N} \cdot \nabla\right.\right. w_{i}^{N} a^{k}+\delta_{i} \cdot \nabla w_{i}^{N} \cdot w_{i}^{N}+w_{i}^{N} \cdot \nabla \delta_{i} \cdot w_{i}^{N} \\
&\left.\left.+\nu \mathbb{D}\left(w_{i}^{N}\right) \mathbb{D}\left(a^{k}\right)\right) d x+\gamma \int_{S_{0}^{i}} w_{i}^{N} \cdot \bar{\tau}_{j} a^{k} \bar{\tau}_{j} d S_{0}^{i}\right\} \\
&=\sum_{i=1}^{3}\left(\sum_{j=1}^{2} \sum_{\sigma=0, i} \int_{S_{\sigma}^{i}} B_{\sigma j}^{i} a^{k} \cdot \bar{\tau}_{j} d S_{\sigma}^{i}+\int_{\Omega_{i}} F_{i} \cdot a^{k} d x\right)
\end{aligned}
$$

for $k=1, \ldots, N$. Thus, $w^{N}$ would be a weak solution to (2.3).

With $(f, g)=\int_{\Omega} f g d x$ and $(f, g)_{S}=\int_{S} f g d S$ this can be rewritten as

$$
\begin{aligned}
\sum_{i=1}^{3}\left\{\left(w_{i, t}^{N}, a^{k}\right)+\left(w_{i}^{N} \cdot \nabla w_{i}^{N}, a^{k}\right)+\left(\delta_{i} \cdot \nabla w_{i}^{N}, a^{k}\right)+\left(w_{i}^{N} \cdot \nabla \delta_{i}, a^{k}\right)\right. & \\
& \left.+\nu\left(\mathbb{D}\left(w_{i}^{N}\right), \mathbb{D}\left(a^{k}\right)\right)+\gamma\left(w_{i}^{N} \cdot \bar{\tau}_{j}, a^{k} \cdot \bar{\tau}_{j}\right)_{S_{0}^{i}}\right\} \\
= & \sum_{i=1}^{3}\left[\sum_{j=1}^{2} \sum_{\sigma=0, i}\left(B_{\sigma j}^{i}, a^{k} \cdot \bar{\tau}_{j}\right)_{S_{\sigma}^{i}}+\left(F_{i}, a^{k}\right)\right], \quad k=1, \ldots, N .
\end{aligned}
$$

Thus,

$$
\begin{aligned}
\left(\frac{d}{d t} w^{N}, a^{k}\right) & +\left(w^{N} \cdot \nabla w^{N}, a^{k}\right)+\left(\delta \cdot \nabla w^{N}, a^{k}\right)+\left(w^{N} \cdot \nabla \delta, a^{k}\right) \\
& +\nu\left(\mathbb{D}\left(w^{N}\right), \mathbb{D}\left(a^{k}\right)\right)+\gamma\left(w^{N} \cdot \bar{\tau}_{j}, a^{k} \cdot \bar{\tau}_{j}\right)_{S_{0}} \\
= & \sum_{i=1}^{3} \sum_{j=1}^{2} \sum_{\sigma=0, i}\left(B_{\sigma j}, a^{k} \cdot \bar{\tau}_{j}\right)_{S_{\sigma}^{i}}+\left(F, a^{k}\right), \quad k=1, \ldots, N .
\end{aligned}
$$

The above equations are in fact a system of ordinary differential equations for the functions $C_{k N}(t)$. The properties of the sequence $a^{k}$ imply

$$
\left|w^{N}(\cdot, t)\right|_{2, \Omega}^{2}=\sum_{k=1}^{N} C_{k N}^{2}(t) .
$$

On the other hand, we can obtain a priori bounds for the approximate solutions $w^{N}$ of the same form as in (2.11):

$$
\begin{aligned}
\left|w^{N}\right|_{V_{2}^{0}\left(\Omega^{T}\right)}^{2} & =\sup _{0 \leq t \leq T}\left|w^{N}\right|_{2, \Omega}+\int_{0}^{T}\left|\nabla w^{N}\right|_{2, \Omega} d t^{\prime} \\
& \leq c e^{c\left(\left|a_{1}\right|_{3,6, S_{1}^{T}}^{6}+|\nabla \alpha|_{3,2, \Omega^{T}}^{2}\right)}\left(\int_{0}^{T} \Gamma^{2}\left(t^{\prime}\right) d t^{\prime}+|w(0)|_{2, \Omega}^{2}\right) \leq C .
\end{aligned}
$$


Therefore, $\sup _{0 \leq t \leq T}\left|C_{k N}(t)\right|$ is bounded on $[0, T]$ and $w^{N}$ are well defined for all times $t$.

Define now $\psi_{N, k} \equiv\left(w^{N}(x, t), a^{k}(x)\right)$. This sequence is uniformly bounded by (3.2). We can also show that it is equicontinuous. Namely, we integrate (3.1) with respect to $t$ from $t$ to $t+\Delta t$ to obtain

$$
\begin{aligned}
\mid \psi_{N, k}( & +\Delta t)-\psi_{N, k}(t) \mid \\
\leq & \sup _{x \in \Omega}\left|a^{k}(x)\right| \int_{t}^{t+\Delta t}\left(\left|w^{N} \cdot \nabla w^{N}\right|_{2, \Omega}+\left|\delta \cdot \nabla w^{N}\right|_{2, \Omega}\left|w^{N} \cdot \nabla \delta\right|_{2, \Omega}+|F|_{2, \Omega}\right) d t^{\prime} \\
& +\nu\left|\nabla a^{k}\right|_{2, \Omega} \int_{t}^{t+\Delta t}\left|\nabla w^{N}\right|_{2, \Omega} d t^{\prime} \\
& +\gamma \sup _{x \in S}\left|a^{k}(x)\right| \int_{t}^{t+\Delta t}\left(\left|w^{N} \cdot \bar{\tau}_{j}\right|_{2, S_{0}}+\sum_{i=1}^{3} \sum_{j=1}^{2} \sum_{\sigma=0, i}\left|B_{\sigma j}\right|_{2, S_{\sigma}^{i}}\right) d t^{\prime} \\
\leq & \sup _{x \in \Omega}\left|a^{k}(x)\right| \sqrt{\Delta t}\left(\sup _{x \in \Omega}\left|w^{N}\right|_{2, \Omega}\left(\left|\nabla w^{N}\right|_{2, \Omega^{T}}+|\nabla \delta|_{2, \Omega^{T}}\right)\right. \\
& \left.+\sup _{x \in \Omega}|\delta|_{2, \Omega}\left|\nabla w^{N}\right|_{2, \Omega^{T}}\right) \\
& +\sup _{x \in \Omega}\left|a^{k}(x)\right| \int_{t}^{t+\Delta t}|F|_{2, \Omega} d t^{\prime}+\nu\left|\nabla a^{k}\right|_{2, \Omega} \sqrt{\Delta t}\left|\nabla w^{N}\right|_{2, \Omega^{T}} \\
& +\gamma \sup _{x \in S}\left|a^{k}(x)\right|\left(\sqrt{\Delta t}\left|\nabla w^{N}\right|_{2, \Omega^{T}}+\int_{t}^{t+\Delta t} \sum_{j=1}^{2}\left|B_{j}\right|_{2, S}\right) d t^{\prime} \\
\leq & C(k)\left(\sqrt{\Delta t}+\int_{t}^{t+\Delta t}\left(|F|_{2, \Omega}+\sum_{j=1}^{2}\left|B_{j}\right|_{2, S}\right) d t^{\prime}\right) .
\end{aligned}
$$

We can see that for given $k$ and $N \geq k$ the r.h.s. tends to zero as $\Delta t \rightarrow 0$ uniformly in $N$. Thus, one can choose a subsequence $N_{m}$ such that $\psi_{N_{m}, k}$ converges as $m \rightarrow \infty$ uniformly to some continuous function $\psi_{k}$ for any given $k$. Since the limit function $w$ is defined as

$$
w(x, t)=\sum_{k=1}^{\infty} \psi_{k}(t) a^{k}(x),
$$

we conclude that $\left(w^{N_{m}}-w, \psi\right)$ tends to zero as $m \rightarrow \infty$ uniformly with respect to $t \in[0, T]$ for any $\psi \in J_{2}^{0}(\Omega)$, and $w(x, t)$ is continuous in $t$ in the weak topology. Moreover, estimate (3.2) remains true for the limit function $w$. 
We will show that $\left\{w^{N_{m}}\right\}$ converges strongly in $L_{2}\left(\Omega^{T}\right)$. To this end, we need to apply the following version of the Friedrichs lemma: for any $\varepsilon>0$, there exists $N_{\varepsilon}$ such that for any $u \in W_{2}^{1}(\Omega)$,

$$
\|u\|_{2, \Omega}^{2} \leq \sum_{k=1}^{N_{\varepsilon}}\left(u, a^{k}\right)+\varepsilon\|\nabla u\|_{2, \Omega}^{2} .
$$

This in terms of $u=w^{N_{m}}-w^{N_{l}}$ reads

$$
\left\|w^{N_{m}}-w^{N_{l}}\right\|_{2, \Omega^{T}}^{2} \leq \sum_{k=1}^{N_{\varepsilon}} \int_{0}^{T}\left(w^{N_{m}}-w^{N_{l}}, a^{k}\right) d t+\varepsilon\left\|\nabla w^{N_{m}}-\nabla w^{N_{l}}\right\|_{2, \Omega^{T}}^{2} .
$$

By (3.2), we have

$$
\left\|\nabla w^{N_{m}}-\nabla w^{N_{l}}\right\|_{2, \Omega^{T}}^{2} \leq 2 C^{2}
$$

for some constant $C$. The above integral, for given $N_{\varepsilon}$, can be arbitrarily small provided $m$ and $l$ are sufficiently large, so it tends to zero as $m, l \rightarrow \infty$. Therefore, $\left\{w^{N_{m}}\right\}$ converges strongly in $L_{2}\left(\Omega^{T}\right)$.

We summarize the above convergence properties of the sequence $\left\{w^{N_{m}}\right\}$ :

(i) $w^{N_{m}} \rightarrow w$ strongly in $L_{2}\left(\Omega^{T}\right)$ for some $w$,

(ii) $w^{N_{m}} \rightarrow w$ weakly in $L_{2}(\Omega)$ uniformly with respect to $t \in[0, T]$,

(iii) $\nabla w^{N_{m}} \rightarrow \nabla w$ weakly in $L_{2}\left(\Omega^{T}\right)$.

For given $\Phi^{k}=\sum_{j=1}^{k} d_{j}(t) a^{j}(x)$, the sequence $\left\{w^{N_{m}}\right\}$ satisfies the identities

$$
\begin{aligned}
& \int_{\Omega}\left(\frac{d}{d t} w^{N_{m}} \Phi^{k}+\left(w^{N_{m}} \cdot \nabla w^{N_{m}}+\delta \cdot \nabla w^{N_{m}}+w^{N_{m}} \cdot \nabla \delta\right) \Phi^{k}+\nu \mathbb{D}\left(w^{N_{m}}\right) \mathbb{D}\left(\Phi^{k}\right)\right) d x \\
& \quad+\gamma \int_{S_{0}} w^{N_{m}} \cdot \bar{\tau}_{j} \Phi^{k} \cdot \bar{\tau}_{j} d S_{0}=\sum_{i=1}^{3} \sum_{j=1}^{2} \sum_{\sigma=0, i} \int_{S_{\sigma}^{i}} B_{\sigma j} \Phi^{k} \cdot \bar{\tau}_{j} d S_{\sigma}^{i}+\int_{\Omega} F \Phi^{k} d x
\end{aligned}
$$

Then we can pass to the limit as $m \rightarrow \infty$ to obtain the identity for $w$. The conditions $\operatorname{div} w^{N}=0,\left.w^{N} \cdot \bar{n}\right|_{S^{T}}=0$ stay true for the limit function $w$ as well.

It remains to consider the $\operatorname{limit}_{\lim _{t \rightarrow 0}} w(x, t)$. We note that the $w^{N_{m}}$ satisfy the relation $(2.12)$ (if we use the test function $w^{N_{m}}$ ). This yields

$$
\left|w^{N_{m}}\right|_{2, \Omega} \leq\left|w_{0}\right|_{2, \Omega}+\int_{0}^{t}\left(|F|_{2, \Omega}+|B|_{2, S}\right) d t^{\prime} .
$$

In the limit $m \rightarrow \infty$ we obtain

$$
|w|_{2, \Omega} \leq\left|w_{0}\right|_{2, \Omega}+\int_{0}^{t}\left(|F|_{2, \Omega}+|B|_{2, S}\right) d t^{\prime}
$$


which implies

$$
\varlimsup_{t \rightarrow 0}|w|_{2, \Omega} \leq\left|w_{0}\right|_{2, \Omega} .
$$

On the other hand, since $w^{N_{m}}$ tends to $w$ as $m \rightarrow \infty$, we have $\left|w^{N_{m}}-w_{0}\right|_{2, \Omega}$ $\rightarrow 0$. Therefore, $\left|w^{N_{m}}-w_{0}\right| \rightarrow 0$ weakly in $L_{2}(\Omega)$ as $t \rightarrow 0$ and

$$
\left|w_{0}\right|_{2, \Omega} \leq \varliminf_{t \rightarrow 0}|w|_{2, \Omega} .
$$

We conclude that the $\operatorname{limit}_{\lim } \rightarrow 0|w|_{2, \Omega}$ exists and is equal to $\left|w_{0}\right|_{2, \Omega}$ where the convergence is strong, in the $L_{2}(\Omega)$ norm.

Consequently, we have proved the following result.

TheOREM 1. Let the assumptions of Lemma 2.3 be satisfied. Then there exists a weak solution $w$ to problem (2.3) such that $w$ is weakly continuous with respect to $t$ in $L_{2}(\Omega)$ norm and $w$ converges to $w_{0}$ as $t \rightarrow 0$ strongly in $L_{2}(\Omega)$ norm.

4. Appendix: sketch of proof of Lemma 2.1. We discuss the properties of the functions $\varphi_{i}, i=1,2,3$, solving problem (2.1). To this end, we need the notion of a regularizer and a partition of unity for the domain $\Omega$. Namely, let us define two collections of open subsets $\left\{\omega^{(k)}\right\}$ and $\left\{\Omega^{(k)}\right\}, k \in \mathcal{M} \cup \mathcal{N}$, such that $\overline{\omega^{(k)}} \subset \Omega^{(k)} \subset \Omega, \bigcup_{k} \omega^{(k)}=\bigcup_{k} \Omega^{(k)}=\Omega, \overline{\Omega^{(k)}} \cap S=\emptyset$ for $k \in \mathcal{M}$ and $\overline{\Omega^{(k)}} \cap S \neq \emptyset$ for $k \in \mathcal{N}$. We assume that at most a finite number of $\Omega^{(k)}$ have nonempty intersection.

We will treat in more detail only the local problem on some sufficiently small subset $\Omega^{N} \subset \Omega$ such that $\Omega^{N} \cap D_{2} \neq \emptyset$ and $\Omega^{N} \cap S_{0}^{i} \neq \emptyset, i=1,2$. The case of a domain that intersects $D_{3}$ is analogous and subsets that lie entirely (i.e. with their closures) in one of $\Omega^{i}, i=1,2,3$, are much easier to treat.

First, we straighten the boundary $\left(S_{0}^{1} \cup S_{0}^{2}\right) \cap \Omega^{N}$ and by the reflection technique we transform the problem on $\Omega^{N}$ to an equivalent problem on some subset $\Omega^{M}$ where $\Omega^{M} \cap D_{2} \neq \emptyset$ and $\operatorname{int}\left\{\Omega^{M}\right\} \cap S_{0}^{i} \neq \emptyset, i=1,2$ (see Figure 2.)

The system (2.1) now reads

$$
\begin{aligned}
& -\Delta \varphi_{1}=\operatorname{div} \alpha_{1} \quad \text { in } \Omega^{M} \cap \Omega_{1}, \\
& -\Delta \varphi_{2}=\operatorname{div} \alpha_{2} \quad \text { in } \Omega^{M} \cap \Omega_{2}, \\
& \left.\frac{\partial \varphi_{1}}{\partial n_{1}}\right|_{D_{2}}=\left.\frac{\partial \varphi_{2}}{\partial n_{1}}\right|_{D_{2}} .
\end{aligned}
$$

Here, we denote in fact by $\varphi$ the new function $\varphi \zeta$ where $\zeta$ is a smooth function with compact support in $\Omega^{M}$. In the new coordinates the local problem on 



Fig. 2. Transformation from $\Omega^{N}$ to $\Omega^{M}$

$\Omega^{M}$ takes the following form in a half-space:

$$
\begin{aligned}
& -\Delta \varphi_{1}=\operatorname{div} \alpha_{1} \quad \text { for } x_{3}>0, \\
& -\Delta \varphi_{2}=\operatorname{div} \alpha_{2} \quad \text { for } x_{3}<0, \\
& \left.\frac{\partial \varphi_{1}}{\partial x_{3}}\right|_{x_{3}=0}=\left.\frac{\partial \varphi_{2}}{\partial x_{3}}\right|_{x_{3}=0},
\end{aligned}
$$

and it is completed with the conditions at infinity:

$$
\begin{aligned}
& \varphi_{1} \rightarrow 0 \quad \text { as } x_{3} \rightarrow \infty, \\
& \varphi_{2} \rightarrow 0 \quad \text { as } x_{3} \rightarrow-\infty .
\end{aligned}
$$

We introduce new functions $u_{i}=\varphi_{i}-\widetilde{\varphi}_{i}$ where $\widetilde{\varphi}_{i}$ satisfy the first two equations of the system (4.1). Therefore, we consider the equivalent problem

$$
\begin{aligned}
& -\Delta u_{1}=0 \quad \text { for } x_{3}>0, \\
& -\Delta u_{2}=0 \quad \text { for } x_{3}<0, \\
& \frac{\partial u_{1}}{\partial x_{3}}-\left.\frac{\partial u_{2}}{\partial x_{3}}\right|_{x_{3}=0}=\frac{\partial \widetilde{\varphi}_{2}}{\partial x_{3}}-\left.\frac{\partial \widetilde{\varphi}_{1}}{\partial x_{3}}\right|_{x_{3}=0} \equiv-\psi_{1}, \\
& u_{1}-\left.u_{2}\right|_{x_{3}=0}=\widetilde{\varphi}_{2}-\widetilde{\varphi}_{1} \equiv \psi_{2}, \\
& u_{1} \rightarrow 0 \quad \text { as } x_{3} \rightarrow \infty \\
& u_{2} \rightarrow 0 \quad \text { as } x_{3} \rightarrow-\infty .
\end{aligned}
$$

Applying the Fourier transform (with respect to $x^{\prime}=\left(x_{1}, x_{2}\right)$ ), i.e.

$$
\widetilde{u}\left(\xi, x_{3}\right)=\int_{\mathbb{R}^{2}} e^{-i \xi x^{\prime}} u\left(x^{\prime}, x_{3}\right) d x^{\prime},
$$


where $\xi=\left(\xi_{1}, \xi_{2}\right)$ and $\xi \cdot x^{\prime}=\xi_{1} x_{1}+\xi_{2} x_{2}$, we obtain the problem

$$
\begin{aligned}
& \xi^{2} \widetilde{u}_{1}-\frac{\partial^{2} \widetilde{u}_{1}}{\partial x_{3}^{2}}=0 \quad \text { for } x_{3}>0, \\
& \xi^{2} \widetilde{u}_{2}-\frac{\partial^{2} \widetilde{u}_{2}}{\partial x_{3}^{2}}=0 \quad \text { for } x_{3}<0, \\
& \frac{\partial \widetilde{u}_{1}}{\partial x_{3}}-\left.\frac{\partial \widetilde{u}_{2}}{\partial x_{3}}\right|_{x_{3}=0}=-\widetilde{\psi}_{1}, \\
& \widetilde{u}_{1}-\left.\widetilde{u}_{2}\right|_{x_{3}=0}=\widetilde{\psi}_{2}, \\
& \widetilde{u}_{1} \rightarrow 0 \quad \text { as } x_{3} \rightarrow \infty, \\
& \widetilde{u}_{2} \rightarrow 0 \quad \text { as } x_{3} \rightarrow-\infty
\end{aligned}
$$

We can easily find the solutions

where

$$
\widetilde{u}_{1}=c_{1} e^{-|\xi| x_{3}}, \quad \widetilde{u}_{2}=c_{2} e^{|\xi| x_{3}},
$$

$$
c_{1}+c_{2}=\widetilde{\psi}_{1}, \quad c_{1}-c_{2}=\widetilde{\psi}_{2},
$$

thus

$$
c_{1}=\frac{1}{2}\left(\widetilde{\psi}_{1}+\widetilde{\psi}_{2}\right), \quad c_{2}=\frac{1}{2}\left(\widetilde{\psi}_{1}-\widetilde{\psi}_{2}\right) .
$$

We want to use $\widetilde{u}_{i}$ to estimate the $H^{2}$ norm of $u_{i}$. By way of example, we examine $\widetilde{u}_{1}$. We observe that

$$
\begin{aligned}
\int_{0}^{\infty}\left|\widetilde{u}_{1}\right|^{2} & =\int_{0}^{\infty} c_{1}^{2} e^{-2|\xi| x_{3}} d x_{3} \leq \frac{c}{|\xi|} \\
\left\|\frac{d}{d x_{3}} \widetilde{u}_{1}\right\|_{L_{2}}^{2} & =\int_{0}^{\infty}\left|\frac{d}{d x_{3}} \widetilde{u}_{1}\right|^{2}=c_{1}^{2} \int_{0}^{\infty}|\xi|^{2} e^{-2|\xi| x_{3}} d x_{3} \leq c|\xi|, \\
\left\|\frac{d^{2}}{d x_{3}^{2}} \widetilde{u}_{1}\right\|_{L_{2}}^{2} & \leq c|\xi|^{3} .
\end{aligned}
$$

Consequently,

$$
\begin{aligned}
\sum_{i=1}^{2}\left\|u_{i}\right\|_{H^{2}}^{2} & =\int\left(\left[\left(1+\xi^{2}\right) \widetilde{u}\right]^{2}+\left|\frac{d^{2}}{d x_{3}^{2}} \widetilde{u}\right|^{2}\right) d \xi \\
& \leq \int\left(\left(1+\xi^{2}\right)^{2} \frac{|\widetilde{\psi}|^{2}}{|\xi|}+|\xi|^{3}|\widetilde{\psi}|^{2}\right) d \xi_{1} d \xi_{2} \\
& \leq c\|\widetilde{\psi}\|_{H^{3 / 2}\left(\mathbb{R}^{2}\right)}^{2} \leq c\|\widetilde{\alpha}\|_{H^{1}\left(\mathbb{R}^{3}\right)} .
\end{aligned}
$$

Hence, by the regularizer technique and the a priori estimate on $\varphi$,

$$
\sum_{i=1}^{3} \int_{\Omega_{i}}\left|\nabla \varphi_{i}\right|^{2} \leq c \sum_{i=1}^{3} \int_{\Omega_{i}}\left|\nabla \alpha_{i}\right|^{2}
$$

we deduce the statement of Lemma 2.1 and the estimate (2.2). 


\section{References}

[L] O. A. Ladyzhenskaya, Mathematical Theory of Viscous Incompressible Flow, Nauka, Moscow 1970 (in Russian).

[Z1] W. M. Zajączkowski, Global regular nonstationary flow for the Navier-Stokes equations in a cylindrical pipe, Topol. Methods Nonlinear Anal. 26 (2005), 221-286.

[Z2] - Global existence of axially symmetric solutions to Navier-Stokes equations with large angular component of velocity, Colloq. Math. 100 (2004), 243-263.

Joanna Rencławowicz

Institute of Mathematics

Polish Academy of Sciences

Śniadeckich 8

00-956 Warszawa, Poland

E-mail: jr@impan.gov.pl
Wojciech M. Zajączkowski

Institute of Mathematics

Polish Academy of Sciences

Śniadeckich 8

00-956 Warszawa, Poland

E-mail: wz@impan.gov.pl

and

Institute of Mathematics and Cryptology

Military University of Technology

Kaliskiego 2

00-908 Warszawa, Poland

Received on 2.3.2006;

revised version on 5.4 .2006 